\title{
Service-outage Capacity Maximization in Cognitive Radio
}

\author{
Athipat Limmanee ${ }^{\dagger}$, Subhrakanti Dey ${ }^{\ddagger}$, and Jamie S. Evans ${ }^{\S}$ \\ Department of Electrical and Electronic Engineering, The University of Melbourne, Australia. \\ email: a.limmanee@pgrad.unimelb.edu.au ${ }^{\dagger},\left\{\right.$ sdey $\left.^{\ddagger}, j^{\S} e^{\S}\right\}$ unimelb.edu.au
}

\begin{abstract}
In spectrum sharing based cognitive radio networks, unlicensed users (secondary users) are allowed to communicate over the same frequency band as the licensed users (primary users) as long as the required quality-of-service $(\mathrm{QoS})$ of the licensed users is guaranteed. This paper focuses on a cognitive radio network, where a secondary user (SU) sharing the same frequency band with a primary user (PU) wishes to transmit delay-insensitive as well as delay-sensitive data while the PU is interested in meeting a minimum rate guarantee for delaysensitive services. Typically, PU's are oblivious to the SU's existence and has its own power policy based on channel side information (CSI) of its direct gain between PU transmitter and PU receiver. Under the assumption that SU knows PU's power policy and CSI of the entire network, we solve the optimal power allocation problem of maximizing SU's ergodic capacity subject to PU's outage probability constraint (POC), SU's outage probability constraint (SOC), and SU's average power constraint. We generalize earlier results which considered either ergodic capacity maximization or outage probability minimization for SU with POC, to the so-called service-outage based capacity optimization for SU with POC. We use a rigorous probabilistic power allocation technique that allows us to derive optimal power policies that are applicable to both continuous and discrete fading channels.
\end{abstract}

\section{INTRODUCTION}

Recently, there has been an explosion of interest in cognitive radio as it offers a potential solution to the problem of spectrum scarcity for wireless applications [1], [2]. The basic rule in cognitive radio environments is that any wireless cognitive devices (secondary users (SU)) can exist in licensed or unlicensed bands if they only minimally disrupt the communications of coexisting noncognitive devices (primary users (PU)). There are three main cognitive radio network paradigms that SUs can use to manage their interferences to PUs: interweave, overlay, and underlay [2].

In this paper, we focus on the 'underlay' paradigm (also known as the spectrum sharing scenario) where SUs can transmit concurrently with PUs by suitably exploiting CSI of the network. However, SUs still have to deal with the fundamental tradeoff between maximizing their own performance, e.g. ergodic capacity, and minimizing the performance loss of the primary network under appropriate power constraints. This fundamental tradeoff has motivated researchers to derive various optimal power schemes that maximize SUs' ergodic capacity under various types of constraints to protect PUs' transmission. The common approach to protect primary users in underlay system is by limiting the peak/average interference power at primary receivers caused by secondary transmitters [3] rather than limiting power at secondary transmitters. With CSI between secondary transmitter and primary receiver assumed at SU, [4] considered a single SU and a single/multiple PU and derived the optimal power allocation scheme to achieve the secondary's ergodic capacity under a peak interference power constraint (PIPC) or an average interference power constraint (AIPC) at the PU receiver in fading channels. [5] studied the same network setup and considered the problems of maximizing SU's ergodic capacity and SU's outage capacity under both PIPC and AIPC. Later, [6] solved similar optimization problems while considering additional transmission power constraints (peak or average) for the SU. With sophisticated techniques recently developed found in [7] and [8], the direct gain between primary user nodes can be revealed to SU. [9] introduced a new constraint called 'primary user ergodic capacity loss constraint' (PLPC). Later, a PU outage probability constraint (POC) was introduced to the problem of maximizing SU's ergodic capacity in [10] and minimizing SU's outage probability in [11] in order to ensure PU maintains a basic rate with a maximum outage probability.

This paper focuses on finding an optimal power allocation policy for maximizing SU's ergodic capacity under an SU average power constraint, SU's outage probability constraint (SOC), and a POC. This problem is related to the "serviceoutage capacity problem", previously studied in [12], in the sense that if POC is discarded, the two problems are exactly the same. Under the assumption that PU has a transmission policy that is based on its own channel between its transmitter and receiver only and that the SU has knowledge of this PU transmission policy as well as the full CSI of the entire network, we solve the SU's service-outage capacity maximization problem with $\mathrm{POC}$ by using a rigorous "probabilistic power allocation" method, first proposed in [13]. This technique allows us to treat the optimal power allocation problem as a convex optimization problem and renders our power allocation results applicable to both continuous as well as discrete fading channels.

The rest of paper is organised as follows. Section II presents the system model. Problem formulation is presented in Section III. The derivation the optimal solution is shown in Section IV. Later, we introduce several related problems from previous works which serve as bounds to our main problem in Section V, prior to showing illustrative numerical results in Section VI. Concluding remarks are presented in Section VII. 
All proofs barring that for the main result (the derivation of optimal probabilistic power allocation policies) are provided in a longer version of this paper available in [14] .

Notation: $\operatorname{Pr}\{\cdot\}$ denotes the probability. $E[\cdot]$ denotes the statistical expectation. $f(\cdot)$ represents a probability density function (PDF), while $F(\cdot)$ indicates the corresponding cumulative distribution function $(\mathrm{CDF}) .(\mathcal{V})^{+}=\max (\mathcal{V}, 0) . l(z)$ denotes the indicator function such that $l(z)$ is 1 when $z$ is true and 0 otherwise. The notation $\frac{\partial y}{\partial x^{*}}$ is the derivative of $y$ with respect to $x$ evaluated at $x=x^{*} . X_{w}$ represents a Bernoulli $w$ random variable, where $X_{w}=1$ with probability $w$ and $X_{w}=0$ with probability $1-w$.

\section{SySTEM MODEL}

We consider a single primary transmitter-receiver pair (PTPR) and a single secondary transmitter-receiver pair (ST-SR) accessing the same frequency band in a typical spectrum sharing scenario. All channels involved in this cognitive radio network are assumed to be independent block fading additive white Gaussian noise (BF-AWGN) channels [13]. The additive noises at PR and SR are assumed to be independent Gaussian random variables with zero mean and variance $N_{0}$. As shown in Figure 1, the instantaneous channel power gains for the link PT-PR, ST-SR, PT-SR, and ST-PR are denoted by $g, h, \alpha$, and $\beta$, respectively. Let $\nu \triangleq\{g, h, \alpha, \beta\}$ denote the combined channel state vector. The vector fading process $\nu$ is assumed to be stationary and ergodic, with a cumulative distribution function $F(\nu)$.

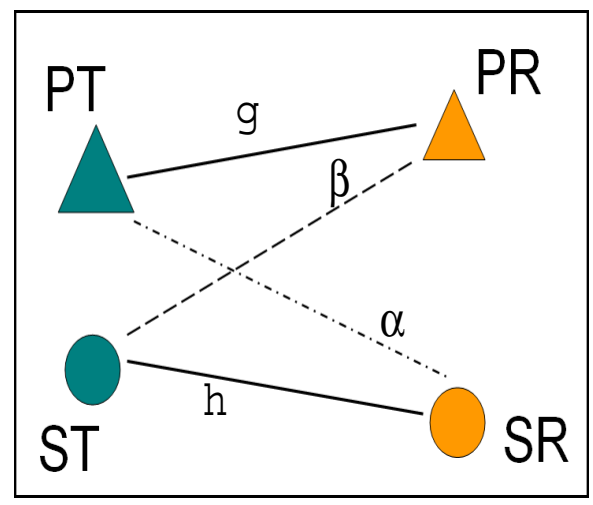

Fig. 1. System model

Let $P_{s}(\nu)$ denote SU's power allocation strategy with respect to the channel state $\nu$ while the instantaneous transmission rates for PU and SU are $r_{p}$ and $r_{s}$, respectively. Thus, the instantaneous rates for $\mathrm{PU}$ and $\mathrm{SU}$ are

$$
\begin{array}{ll}
r_{p}\left(\nu, P_{s}(\nu)\right)= & \log \left(1+\frac{g P_{p}(g)}{\beta P_{s}(\nu)+N_{0}}\right) \\
r_{s}\left(\nu, P_{s}(\nu)\right)= & \log \left(1+\frac{h P_{s}(\nu)}{\alpha P_{p}(g)+N_{0}}\right)
\end{array}
$$

Note that we drop the constant $\frac{1}{2}$ in the instantaneous rate expressions above and use natural logarithm for simplicity. Let $r_{p}^{0}$ and $r_{s}^{0}$ denote PU's and SU's service rates. Further, observe that we use $P_{p}(g)$ to represent PU's power strategy. Note that PU's statergy is assumed to be determined by the direct channel power gain $g$ between PT and PR only and it can be constant power control or standard water-filling [15], i.e. PU's power control is independent on the existence of SU. However, SU is assumed to know the PU's transmission power scheme, the channel state vector $\nu$, and therefore $P_{p}(g)$.

In this paper, we assume that PU adopts an 'ON-OFF' power control, transmitting with constant power $P_{p}(g)=P_{c}$ when $g<g_{T}=\frac{\left(e^{r_{p}^{0}}-1\right) N_{0}}{P_{c}}$, that is, when PU will be in outage even when SU is not operating. Therefore PT is 'ON' only when it sees $g \geq g_{T}$. Thus, $P_{p}(g)=P_{c}$ when $g \geq g_{T}$, otherwise $P_{p}(g)=0$. The primary outage probability when $\mathrm{SU}$ is not operating is given by $\epsilon_{p}^{c}=\operatorname{Pr}\left\{\log \left(1+\frac{g P_{c}}{N_{0}}\right)<r_{p}^{0}\right\}$. Once SU is active. The transmission outage probability of PU becomes $\epsilon_{p}^{\prime}=\operatorname{Pr}\left\{\log \left(1+\frac{g P_{c}}{\beta P_{s}(\nu)+N_{0}}\right)<r_{p}^{0}\right\}$. Therefore, we can avoid unacceptable performance loss for PU by limiting the total outage probability $\epsilon_{p}$ such that $\epsilon_{p}^{c}+\epsilon_{p}^{\prime} \leq \epsilon_{p}$.

\section{PRoblem Formulation}

The SU power allocation problem is to maximize the secondary ergodic capacity subject to the average secondary user's power constraint and the constraints that the PU's and SU's outage probabilities must not be more than $\epsilon_{p}$ and $\epsilon_{s}$, respectively. We can now formulate our problem (P1) as follows.

(P1)

$$
\begin{array}{ll}
\max _{P_{s}(\nu)} & R_{s}=E\left[r_{s}\left(\nu, P_{s}(\nu)\right)\right] \\
\text { s.t. } & \operatorname{Pr}\left\{r_{p}\left(\nu, P_{s}(\nu)\right)<r_{p}^{0}\right\} \leq \epsilon_{p} \\
& \operatorname{Pr}\left\{r_{s}\left(\nu, P_{s}(\nu)\right)<r_{s}^{0}\right\} \leq \epsilon_{s} \\
& E\left[P_{s}(\nu)\right] \leq P_{a v} \\
& P_{s}(\nu) \geq 0
\end{array}
$$

It was shown in [16] and [13] that for outage capacity based optimization problem, a deterministic power allocation policy may not be optimal for discrete fading channel distributions. Hence, we will solve (P1) by using a probabilistic power allocation technique, which also allows us to formulate the above problem as a convex optimization problem [16]. Let $P_{s}(\nu)$ in $(\mathbf{P 1})$ indicate a probabilistic power allocation scheme with conditional PDF $f_{P_{s} \mid \nu}\left(p_{s} \mid \nu\right)$ whereas $p_{s}(\nu)$ represents a deterministic scheme. The power allocation only depends on the instantaneous channel state vector $\nu$ when ergodicity and perfect CSI are assumed. Thus,

$$
\begin{aligned}
& E\left[r_{s}\left(\nu, P_{s}(\nu)\right)\right]=\iint r_{s}\left(\nu, p_{s}(\nu)\right) f_{P_{s} \mid \nu}\left(p_{s} \mid \nu\right) d p_{s}(\nu) d F(\nu) \\
& E\left[P_{s}(\nu)\right]=\iint p_{s}(\nu) f_{P_{s} \mid \nu}\left(p_{s} \mid \nu\right) d p_{s}(\nu) d F(\nu) \\
& \operatorname{Pr}\left\{r_{s}\left(\nu, P_{s}(\nu)\right)<r_{s}^{0}\right\} \\
& \quad=\iiint 1\left(r_{s}\left(\nu, p_{s}(\nu)\right)<r_{s}^{0}\right) f_{P_{s} \mid \nu}\left(p_{s} \mid \nu\right) d p_{s}(\nu) d F(\nu) \\
& \operatorname{Pr}\left\{r_{p}\left(\nu, P_{s}(\nu)\right)<r_{p}^{0}\right\} \\
& \quad=\iint 1\left(r_{p}\left(\nu, p_{s}(\nu)\right)<r_{p}^{0}\right) f_{P_{s} \mid \nu}\left(p_{s} \mid \nu\right) d p_{s}(\nu) d F(\nu)
\end{aligned}
$$

where $f_{P_{s} \mid \nu}\left(p_{s} \mid \nu\right) \geq 0$ satisfies $\int f_{P_{s} \mid \nu}\left(p_{s} \mid \nu\right) d p_{s}(\nu)=1$.

We can now show that there must exist an optimum power allocation $P_{s}(\nu)$ which is randomized between four 
deterministic power allocation schemes defined in (4), and corresponding weighting functions defined in (5).

$$
\begin{aligned}
& p_{1}(\nu)=E\left[P_{s}(\nu) \mid r_{p}\left(\nu, p_{s}(\nu)\right) \geq r_{p}^{0}, r_{s}\left(\nu, p_{s}(\nu)\right) \geq r_{s}^{0}, \nu\right] \\
& p_{2}(\nu)=E\left[P_{s}(\nu) \mid r_{p}\left(\nu, p_{s}(\nu)\right) \geq r_{p}^{0}, r_{s}\left(\nu, p_{s}(\nu)\right)<r_{s}^{0}, \nu\right] \\
& p_{3}(\nu)=E\left[P_{s}(\nu) \mid r_{p}\left(\nu, p_{s}(\nu)\right)<r_{p}^{0}, r_{s}\left(\nu, p_{s}(\nu)\right) \geq r_{s}^{0}, \nu\right] \\
& p_{4}(\nu)=E\left[P_{s}(\nu) \mid r_{p}\left(\nu, p_{s}(\nu)\right)<r_{p}^{0}, r_{s}\left(\nu, p_{s}(\nu)\right)<r_{s}^{0}, \nu\right]
\end{aligned}
$$

$$
\left.\begin{array}{l}
w_{1}(\nu)=\operatorname{Pr}\left\{r_{p}\left(\nu, P_{s}(\nu)\right) \geq r_{p}^{0}, r_{s}\left(\nu, P_{s}(\nu)\right) \geq r_{s}^{0} \mid \nu\right\} \\
w_{2}(\nu)=\operatorname{Pr}\left\{r_{p}\left(\nu, P_{s}(\nu)\right) \geq r_{p}^{0}, r_{s}\left(\nu, P_{s}(\nu)\right)<r_{s}^{0} \mid \nu\right. \\
w_{3}(\nu)=\operatorname{Pr}\left\{r_{p}\left(\nu, P_{s}(\nu)\right)<r_{p}^{0}, r_{s}\left(\nu, P_{s}(\nu)\right) \geq r_{s}^{0} \mid \nu\right. \\
w_{4}(\nu)=\operatorname{Pr}\left\{r_{p}\left(\nu, P_{s}(\nu)\right)<r_{p}^{0}, r_{s}\left(\nu, P_{s}(\nu)\right)<r_{s}^{0} \mid \nu\right\}
\end{array}\right\}
$$

For simplicity, define $p_{r p}(\nu)=\frac{1}{\beta}\left(\frac{g P_{p}(g(\nu))}{e^{r 0} p_{p}^{0}}-N_{0}\right)^{+}$, and $p_{r s}(\nu)=\left(\frac{e^{r_{s}^{0}}-1}{h}\left(\alpha P_{p}(g(\nu))+N_{0}\right)\right)$ as follows.

With the above definitions, we have the following Lemma:

Lemma 1: There exists an optimum solution of problem (2) is expressed by $P_{s}^{*}(\nu)=\sum_{k=1}^{4} X_{w_{k}^{*}}(\nu) p_{k}^{*}(\nu)$, where

$E\left[w_{1}(\nu)+w_{2}(\nu)\right] \geq 1-\epsilon_{p}, \quad E\left[w_{1}(\nu)+w_{3}(\nu)\right] \geq 1-\epsilon_{s}$, $w_{1}(\nu)\left[p_{r p}(\nu)-p_{1}(\nu)\right]+w_{2}(\nu)\left[p_{r p}(\nu)-p_{2}(\nu)\right] \geq 0$, $w_{1}(\nu)\left[p_{1}(\nu)-p_{r s}(\nu)\right]+w_{3}(\nu)\left[p_{3}(\nu)-p_{r s}(\nu)\right] \geq 0$, $\sum_{k=1}^{4} w_{k}(\nu)=1, \quad E\left[P_{s}^{*}(\nu)\right] \leq P_{a v}$.

The interpretation of Lemma 1 is that $P_{s}^{*}(\nu)=p_{k}^{*}(\nu)$ with probability $w_{k}^{*}(\nu)$ while the conditions in Lemma 1 ensure that the probabilistic policy satisfies the POC, SOC and SU average power constraints. By using Lemma 1, we can rewrite (2) as (6)

$$
\begin{array}{cc}
\max _{p_{k}(\nu), w_{k}(\nu)} & E\left[\sum_{k=1}^{4} w_{k}(\nu) r_{s}\left(\nu, p_{k}(\nu)\right)\right] \\
\text { s.t. } & E\left[\sum_{k=1}^{4} w_{k}(\nu) p_{k}(\nu)\right] \leq P_{a v} \\
& E\left[w_{1}(\nu)+w_{2}(\nu)\right] \geq 1-\epsilon_{p} \\
& E\left[w_{1}(\nu)+w_{3}(\nu)\right] \geq 1-\epsilon_{s} \\
& w_{1}(\nu)\left[p_{r p}(\nu)-p_{1}(\nu)\right]+ \\
& w_{2}(\nu)\left[p_{r p}(\nu)-p_{2}(\nu)\right] \geq 0 \\
& w_{1}(\nu)\left[p_{1}(\nu)-p_{r s}(\nu)\right]+ \\
& w_{3}(\nu)\left[p_{3}(\nu)-p_{r s}(\nu)\right] \geq 0 \\
& 1-\sum_{k=1}^{4} w_{k}(\nu)=0 \\
& p_{k}(\nu) \geq 0 \quad, k=1,2,3,4 \\
& w_{k}(\nu) \geq 0 \quad, k=1,2,3,4
\end{array}
$$

We can show that (6) is a convex problem by using Proposition 3 in [16].

\section{DeRIVATION OF OPTIMAL POWER ALLOCATION POLICIES}

We adopt a functional optimization technique similar to [16] and define the following Lagrangian

$$
\begin{aligned}
l\left(\nu, p_{k}(\nu), w_{k}(\nu), \lambda, s_{1}, s_{2}, q(\nu), u(\nu)\right) \\
=w_{1}(\nu)\left(r_{s}\left(\nu, p_{1}(\nu)\right)-\lambda p_{1}(\nu)+s_{1}+s_{2}-\Gamma(\nu)\right)+ \\
\quad w_{2}(\nu)\left(r_{s}\left(\nu, p_{2}(\nu)\right)-\lambda p_{2}(\nu)+s_{1}-\Gamma(\nu)\right)+ \\
\quad w_{3}(\nu)\left(r_{s}\left(\nu, p_{3}(\nu)\right)-\lambda p_{3}(\nu)+s_{2}-\Gamma(\nu)\right)+ \\
\quad w_{4}(\nu)\left(r_{s}\left(\nu, p_{4}(\nu)\right)-\lambda p_{4}(\nu)-\Gamma(\nu)\right)+\Gamma(\nu) \\
\quad w_{1}(\nu)\left[q(\nu)\left[p_{r p}(\nu)-p_{1}(\nu)\right]+u(\nu)\left[p_{1}(\nu)-p_{r s}(\nu)\right]\right]+ \\
\quad w_{2}(\nu) q(\nu)\left[p_{r p}(\nu)-p_{2}(\nu)\right]+w_{3}(\nu)\left[u(\nu)\left[p_{3}(\nu)-p_{r s}(\nu)\right]\right]
\end{aligned}
$$

where $\lambda, s_{1}, s_{2}, q(\nu), u(\nu)$ and $\Gamma(\nu)$ denote the Lagrange multipliers for the constraints $(6 \mathrm{~b})$ to $(6 \mathrm{~g})$. Then, using the generalised Karush-Kuhn-Tucker (KKT) conditions for functional optimization, we have

$$
\begin{aligned}
& \frac{\partial l(\ldots)}{\partial p_{k}^{*}(\nu)} \begin{cases}=0, & p_{k}^{*}(\nu)>0 \\
\leq 0, & p_{k}^{*}(\nu)=0\end{cases} \\
& \frac{\partial l(\ldots)}{\partial w_{k}^{*}(\nu)} \begin{cases}=0, & w_{k}^{*}(\nu)>0 \\
\leq 0, & w_{k}^{*}(\nu)=0\end{cases} \\
& \lambda^{*}\left(E\left[\sum_{k=1}^{4} w_{k}^{*}(\nu) p_{k}^{*}(\nu)\right]-P_{a v}\right)=0 \\
& s_{1}^{*}\left(E\left[w_{1}^{*}(\nu)+w_{2}^{*}(\nu)\right]-\left(1-\epsilon_{p}\right)\right)=0 \\
& s_{2}^{*}\left(E\left[w_{1}^{*}(\nu)+w_{3}^{*}(\nu)\right]-\left(1-\epsilon_{s}\right)\right)=0 \\
& q^{*}(\nu)\left[w_{1}^{*}(\nu)\left(p_{1}^{*}(\nu)-p_{r p}(\nu)\right)+w_{2}^{*}(\nu)\left(p_{2}^{*}(\nu)-p_{r p}(\nu)\right)\right]=0
\end{aligned}
$$

$u^{*}(\nu)\left[w_{1}^{*}(\nu)\left(p_{r s}(\nu)-p_{1}^{*}(\nu)\right)+w_{3}^{*}(\nu)\left(p_{r s}(\nu)-p_{3}^{*}(\nu)\right)\right]=0$

$$
\begin{gathered}
\Gamma^{*}(\nu)\left(1-\sum_{k=1}^{4} w_{k}^{*}(\nu)\right)=0 \\
p_{k}^{*}(\nu) \geq 0, k=1,2,3,4 \\
w_{k}^{*}(\nu) \geq 0, k=1,2,3,4
\end{gathered}
$$

From (7), we also define the following 'benefit functions' corresponding to each $p_{k}$ :

$$
\begin{array}{ll}
\mathcal{B}_{1}\left(\nu, p_{1}^{*}(\nu), \lambda^{*}, s_{1}^{*}, s_{2}^{*}\right) & \triangleq r_{s}\left(\nu, p_{1}^{*}(\nu)\right)-\lambda^{*} p_{1}^{*}(\nu)+s_{1}^{*}+s_{2}^{*} \\
\mathcal{B}_{2}\left(\nu, p_{2}^{*}(\nu), \lambda^{*}, s_{1}^{*}\right) & \triangleq r_{s}\left(\nu, p_{2}^{*}(\nu)\right)-\lambda^{*} p_{2}^{*}(\nu)+s_{1}^{*} \\
\mathcal{B}_{3}\left(\nu, p_{3}^{*}(\nu), \lambda^{*}, s_{2}^{*}\right) & \triangleq r_{s}\left(\nu, p_{3}^{*}(\nu)\right)-\lambda^{*} p_{3}^{*}(\nu)+s_{2}^{*} \\
\mathcal{B}_{4}\left(\nu, p_{4}^{*}(\nu), \lambda^{*}\right) & \triangleq r_{s}\left(\nu, p_{4}^{*}(\nu)\right)-\lambda^{*} p_{4}^{*}(\nu)
\end{array}
$$

For simplicity, we use $\mathcal{B}_{k, \nu}$ as the shorthand notation of $\mathcal{B}_{k}(\ldots)$ for $k=\{1,2,3,4\}$ from now on.

The physical interpretation of (18) is that they denote the return benefit after using power policy $p_{k}^{*}$. For example, if we use $p_{1}^{*}$, the power expense is $\lambda^{*} p_{1}^{*}(\nu)$ and the return is $r_{s}\left(\nu, p_{1}^{*}(\nu)\right)+s_{1}^{*}+s_{2}^{*}$. For simplicity, we define $p_{w f}\left(\nu, \lambda^{*}\right)=\left(\frac{1}{\lambda^{*}}-\frac{\alpha P_{p}(g(\nu))+N_{0}}{h}\right)^{+}$. In the Appendix, we provide the solutions for the four power control schemes and their corresponding weighting functions. 
Feasibility of the main problem: the feasibility of the main problem is directly related to SU's $\epsilon_{s}$-outage capacity with POC whose optimal solution is provided in [11]. Let $C_{\epsilon_{s}}\left(P_{a v}, r_{p}^{0}, \epsilon_{p}\right)$ stand for SU's $\epsilon_{s}$-outage capacity with POC given fixed $r_{p}^{0}, \epsilon_{p}, \epsilon_{s}$, and $\mathrm{SU}$ average power $P_{a v}$. $C_{\epsilon_{s}}\left(P_{a v}, r_{p}^{0}, \epsilon_{p}\right)$ is the maximum instantaneous rate which SU can transmit with an outage probability $\epsilon_{s}$ under POC. So, this problem is feasible iff the SU target rate $r_{s}^{0} \leq C_{\epsilon_{s}}\left(P_{a v}, r_{p}^{0}, \epsilon_{p}\right)$. For simplicity, let $P_{\min }\left(r_{s}^{0}, \epsilon_{s}, r_{p}^{0}, \epsilon_{p}\right)$ be the minimum SU average power needed to support the SU target rate $r_{s}^{0}$ with outage probability $\epsilon_{s}$ and still satisfy POC with $r_{p}^{0}$ and $\epsilon_{p}$. So, the feasibility condition for our main problem is then $P_{a v} \geq$ $P_{\min }\left(r_{s}^{0}, \epsilon_{s}, r_{p}^{0}, \epsilon_{p}\right)$. Clearly, when $P_{a v}=P_{\min }\left(r_{s}^{0}, \epsilon_{s}, r_{p}^{0}, \epsilon_{p}\right)$, SU has to use $p_{1}^{*}(\nu)=p_{3}^{*}(\nu)=p_{r s}(\nu)$ to support its basic rate requirement with outage probability $\epsilon_{s}$ and $p_{2}^{*}(\nu)=$ $p_{4}^{*}(\nu)=0$ when SU is in outage. Details of how to compute $P_{\min }\left(r_{s}^{0}, \epsilon_{s}, r_{p}^{0}, \epsilon_{p}\right)$ are provided in [14] .

With the expressions for the optimal power policies and weighting functions derived in the Appendix, we have the main result of this paper in the following Theorem:

Theorem 1: If $P_{a v}=P_{\min }\left(r_{s}^{0}, \epsilon_{s}, r_{p}^{0}, \epsilon_{p}\right)$, the optimal solution is $P_{s}^{*}(\nu)=\left(X_{w_{1}^{*}}(\nu)+X_{w_{3}^{*}}(\nu)\right) p_{r s}(\nu)$. If $P_{a v}>$ $P_{\min }\left(r_{s}^{0}, \epsilon_{s}, r_{p}^{0}, \epsilon_{p}\right)$, the optimal solution is

$$
\begin{aligned}
& P_{s}^{*}(\nu) \\
& =X_{w_{1}^{*}}(\nu)\left(\left[p_{r s}(\nu)-p_{w f}\left(\nu, \lambda^{*}\right)\right]^{+}-\left[p_{w f}\left(\nu, \lambda^{*}\right)-p_{r p}(\nu)\right]^{+}\right) \\
& +X_{w_{2}^{*}}(\nu)\left(-\left[p_{w f}\left(\nu, \lambda^{*}\right)-p_{r p}(\nu)\right]^{+}\right) \\
& +X_{w_{3}^{*}}(\nu)\left(\left[p_{r s}(\nu)-p_{w f}\left(\nu, \lambda^{*}\right)\right]^{+}\right)+p_{w f}\left(\nu, \lambda^{*}\right)
\end{aligned}
$$

where

$$
w_{i}^{*}(\nu)= \begin{cases}1, & \mathcal{B}_{i, \nu}>\max _{i \neq j} \mathcal{B}_{j, \nu} \\ \kappa_{i}^{*}(\nu), & \mathcal{B}_{i, \nu}=\max _{i \neq j} \mathcal{B}_{j, \nu} \\ 0, & \mathcal{B}_{i, \nu}<\max _{i \neq j} \mathcal{B}_{j, \nu}\end{cases}
$$

where $i, j \in\{1,2,3,4\}, i \neq j, 0<\kappa_{i}^{*}(\nu)<1$, and $\lambda^{*}, s_{1}^{*}$, and $s_{2}^{*}$ are the solutions to

$$
\begin{array}{ll}
E\left[P_{s}^{*}(\nu)\right]=P_{a v}, & E\left[w_{1}^{*}(\nu)+w_{2}^{*}(\nu)\right] \geq 1-\epsilon_{p}, \\
E\left[w_{1}^{*}(\nu)+w_{3}^{*}(\nu)\right] \geq 1-\epsilon_{s}, & \sum_{i=1}^{4} w_{i}^{*}(\nu)=1 .
\end{array}
$$

For continuous fading distributions, it can be easily shown that there exists only one $w_{k}^{*}(\nu)=1$. Also, the optimal $p_{k}^{*}(\nu)$ can be shown to be one of the three deterministic schemes $p_{w f}\left(\nu, \lambda^{*}\right), p_{r p}(\nu)$ and $p_{r s}(\nu)$. So for a given $\nu$, there are at most three of $w_{k}^{*}(\nu)$ and at least one $w_{k}^{*}(\nu)$ that can be considered to be 1 . We will use these facts to search for the solution for $w_{k}^{*}(\nu)$.

Remark 1: Table I summarises all possible cases for continuous fading distributions including the associated power allocation policies, and the candidate non-negative $w_{k}^{*}(\nu)$.

To illustrate, we explain the first two cases only. In the first case, the optimal power policy is just $p_{1}^{*}(\nu)=p_{w f}\left(\nu, \lambda^{*}\right)$ because $p_{r s}(\nu)<p_{w f}\left(\nu, \lambda^{*}\right)$ and $p_{w f}\left(\nu, \lambda^{*}\right)<p_{r p}(\nu)$, so there is only $w_{1}^{*}(\nu)=1$. For the second case, the optimal
TABLE I

SIX POSSIBLE CASES FOR A GIVEN CONTINUOUS FADING CHANNEL STATE $\nu$

\begin{tabular}{lll}
\hline \hline Cases & Power allocation policy & $\begin{array}{c}\text { Candidate } \\
\text { non-negative } w_{k}\end{array}$ \\
\hline$p_{r s}<p_{w f}<p_{r p}$ & $p_{1}^{*}=p_{w f}$ & $w_{1}^{*}$ (always 1$)$ \\
\hline$p_{r p}<p_{w f}<p_{r s}$ & $\begin{array}{l}p_{2}^{*}=p_{r p}, p_{4}^{*}=p_{w f}, \\
p_{3}^{*}=p_{r s}\end{array}$ & $w_{2}^{*}, w_{3}^{*}, w_{4}^{*}$ \\
\hline$p_{w f}<p_{r p}<p_{r s}$ & $p_{2}^{*}=p_{w f}, p_{3}^{*}=p_{r s}$ & $w_{2}^{*}, w_{3}^{*}$ \\
\hline$p_{r p}<p_{r s}<p_{w f}$ & $p_{2}^{*}=p_{r p}, p_{3}^{*}=p_{w f}$ & $w_{2}^{*}, w_{3}^{*}$ \\
\hline$p_{r s}<p_{r p}<p_{w f}$ & $p_{1}^{*}=p_{r p}, p_{3}^{*}=p_{w f}$ & $w_{1}^{*}, w_{3}^{*}$ \\
\hline$p_{w f}<p_{r s}<p_{r p}$ & $p_{2}^{*}=p_{w f}, p_{1}^{*}=p_{r s}$ & $w_{1}^{*}, w_{2}^{*}$ \\
\hline \hline
\end{tabular}

power policies must be determined from all three deterministic policies $p_{w f}\left(\nu, \lambda^{*}\right), p_{r p}(\nu)$ and $p_{r s}(\nu)$ which requires us to consider three candidate non-negative weighting functions from $w_{k}^{*}(\nu), k=1,2,3,4$.

Remark 2: When $P_{p}(g)=0$, candidate non-negative weighting functions are just $w_{3}^{*}(\nu)$ and $w_{4}^{*}(\nu)$ and the solution to the optimal SU power allocation policy is the same as the service outage based solution provided in [16].

\section{BOUNDS}

To strengthen our main result, we also include the ergodic and outage capacity results for the following related problems into our numerical results.

- (P2) : Maximize SU ergodic capacity subject to SU average power constraint.

- (P3) : Maximize SU ergodic capacity subject to SU average power constraint and POC.

- (P4) : Maximize SU ergodic capacity subject to SU average power constraint and SOC.

- (P5) : Maximize SU outage capacity subject to SU average power constraint and POC.

Obviously, when we plot SU ergodic capacity derived as a solution to Problem (P1) against SU average power, (P2) - (P4) provide us upper bounds and (P5) provides a lower bound for $(\mathbf{P 1})$. The solution to $(\mathbf{P} 2)$ is clearly water-filling, while solutions to (P3) and (P5) are provided in [10] and [11], respectively, while solution to (P4) is provided in [16].

\section{NumericAl RESUlts}

In this section, numerical results are illustrated to evaluate the performance of the proposed power allocation policy. All channel gains involved are assumed to be Rayleigh fading, and their channel power gains are thus exponentially distributed with unit mean. Noises at PR and SR are presumed to be equal and AWGN with unit variance, i.e. $N_{0}=1$. The transmit power $P_{c}$ of the $\mathrm{PU}$ is $15 \mathrm{~dB}$ when it is active.

We will use Fig.2 and Fig.3 to illustrate the ergodic capacity performance as a solution to Problem (P1). We set $r_{p}^{0}=0.1$ and $r_{s}^{0}=0.4$ with outage probability thresholds $\epsilon_{p}=\epsilon_{s}=$ 0.1 . In Fig.2, it shows SU ergodic capacity plotted against given SU average power budget $P_{a v}$ corresponding to our main problem (P1) and the other related bounds as mentioned in Section V. In Fig.3, it shows how outage probabilities of PU and SU will behave when the optimal power policy of (P1) 
is applied. As expected, there is a minimum average power $P_{\min }$ required to make $(\mathbf{P 1})$ feasible, i.e. the capacity graph starts exactly at $\mathrm{SU}$ average rate $R_{s}=r_{s}^{0}\left(1-\epsilon_{s}\right)$ in Fig.2, while the primary user outage probability remains less than $\epsilon_{p}$. In other words, it coincides with the solution to problem (P5) at the initial point. With $P_{a v}>P_{\min }$, the SU average rate in (P1) is exactly the same as in (P4) as long as POC is inactive and $\mathrm{SU}$ outage probability equals $\epsilon_{s}$, i.e. it is in 'Stage 1 ' as illustrated in Fig.3. Once both POC and SOC are active, our problem is in 'Stage 2' where our main problem cannot achieve SU average rate as high as the three upper bounds. However, ergodic capacity achieved via (P1) tends to approach the graph of (P3) with increasing power budget. Finally, they become exactly the same in 'Stage 3' where POC is active while SOC is not. Note also that although as SU's average power budget increases, it cannot increase its ergodic capacity as much as achieved via (P2) and (P4) in 'Stage 3' because POC is now active. However, ergodic capacities achieved via (P1) and (P3) are still increasing with increasing $P_{a v}$ since SU can put more power into the good channels using water-filling when PU is not active (i.e, $g<g_{T}$ ).

In Fig.4, PU target rate is varied over three different values and there are two results we need to emphasize. The first effect, denoted by the dotted oval, is that the minimum average power is higher when $r_{p}^{0}$ increases (for a fixed outage probability threshold $\epsilon_{p}$ ). Similarly, a higher PU target rate makes SU ergodic capacity substantially lower than the upper bound achieved via (P3) (for a fixed $P_{a v}$ ).

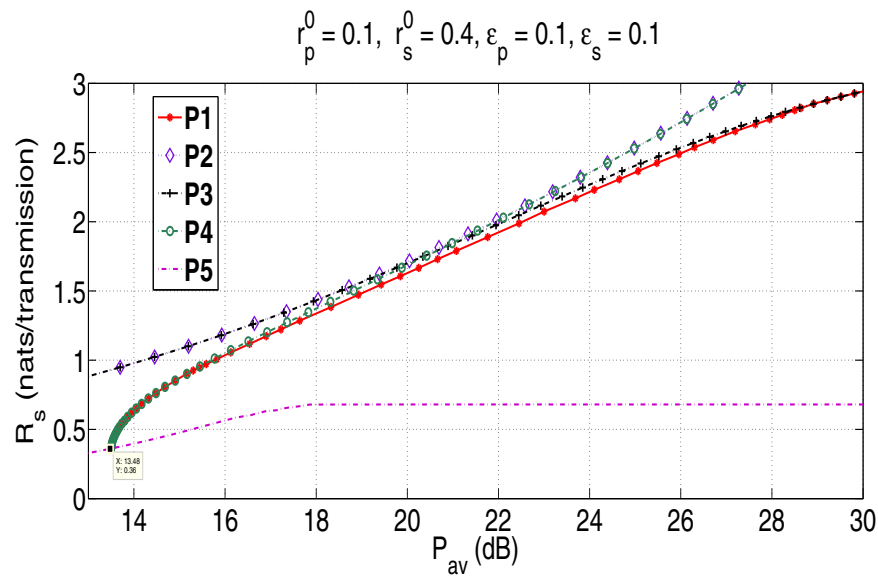

Fig. 2. Average SU rate performance of optimal power allocation for (P1) compared to the bounds $\left(\epsilon_{p}=0.1, \epsilon_{s}=0.1, r_{p}^{0}=0.1, r_{s}^{0}=0.4\right)$

\section{CONCLUSION}

In this paper, we derived an optimal power allocation policy (under full CSI at the SU transmitter and receiver) to achieve maximum SU ergodic capacity in spectrum sharing cognitive radio network over fading channels, under an average SU transmit power constraint and both SU and PU outage probability constraints, which is suitable for scenarios where PU needs to have guaranteed delay-limited performance and SU

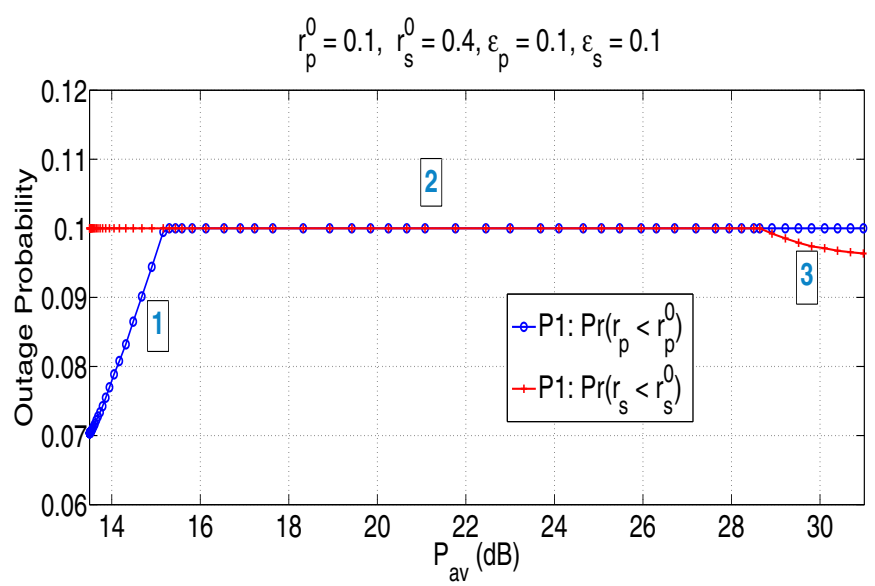

Fig. 3. Outage probability for PU and $\mathrm{SU}$ from $(\mathbf{P 1})\left(\epsilon_{p}=0.1, \epsilon_{s}=\right.$ $0.1, r_{p}^{0}=0.1, r_{s}^{0}=0.4$ )

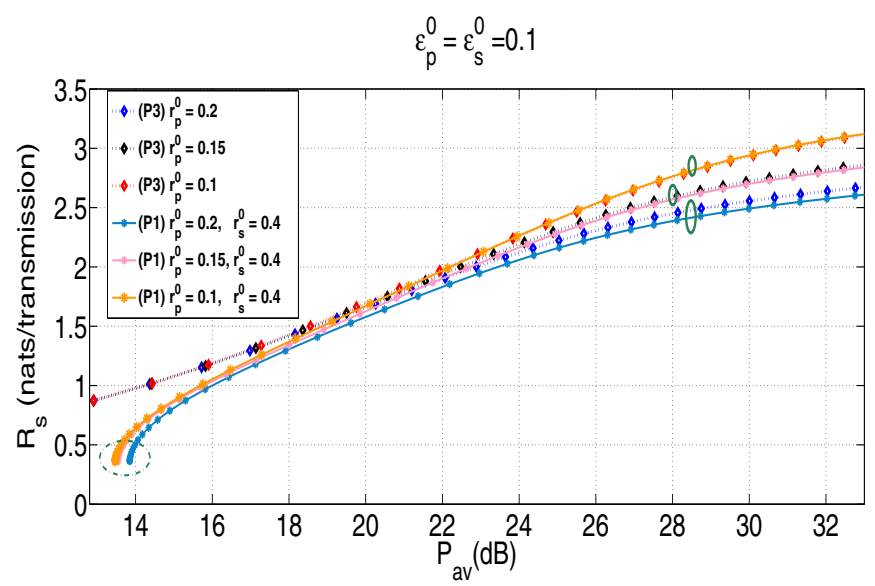

Fig. 4. SU average rate performance with varying $r_{p}^{0}$ with fixed $r_{s}^{0}=0.4$ $\left(\epsilon_{p}=\epsilon_{s}=0.1\right)$

has both delay-sensitive (such as voice and video) and delayinsensitive (such as packetised data) information to transmit. In this work, the primary user are assumed to adapt its power based on its perfect CSI on its direct channel gain between its transmitter and receiver. The optimal solution is derived by using a probabilistic power allocation technique that timeshares among three particular deterministic power allocation policies, thus making this technique more general and applicable to discrete fading distributions. Numerical results illustrate that the proposed power policy can guarantee both PU's and SU's service rate while keeping their outage probability values less than or equal to the predefined values.

\section{APPENDIX}

Derivation of the optimal solutions $p_{k}^{*}(\nu)$ and $w_{k}^{*}(\nu)$

From (8), we can solve for $p_{k}^{*}(\nu)$ where $k \in\{1,2,3,4\}$. Due to space limitation, only the proof for $p_{1}^{*}(\nu)$ is shown whereas only the solutions for $p_{2}^{*}(\nu)$ to $p_{4}^{*}(\nu)$ are provided. So, let $A_{k}(\nu)=\frac{\partial l(\ldots)}{\partial p_{k}^{*}(\nu)}$ and $Z_{k}(\nu)=\frac{\partial r_{s}\left(\nu, p_{k}^{*}(\nu)\right)}{\partial p_{k}^{*}(\nu)}$. Hence, $A_{1}(\nu)=w_{1}^{*}(\nu)\left[Z_{1}(\nu)-\lambda^{*}-q^{*}(\nu)+u^{*}(\nu)\right]$ 
Obviuosly, the solution for $p_{1}^{*}(\nu)$ becomes meaningful if $w_{1}^{*}(\nu)>0$. Therefore, first assuming that $w_{1}^{*}(\nu)>0$, the solution for $p_{1}^{*}(\nu)$ is shown below:

$$
p_{1}^{*}(\nu)=\left\{\begin{array}{cl}
\left(\frac{1}{\lambda^{*}}-\frac{\alpha P_{p}(g(\nu))+N_{0}}{h}\right)^{+}, & w_{1}^{*}(\nu)>0, \\
& q^{*}(\nu)=0 \\
& u^{*}(\nu)=0 \\
\frac{1}{\beta}\left(\frac{g P_{p}(g(\nu))}{e^{r 0}-1}-N_{0}\right)^{+}, & w_{1}^{*}(\nu)>0 \\
& q^{*}(\nu)>0 \\
& u^{*}(\nu)=0 \\
\left(\frac{e_{s}^{r_{s}^{0}}-1}{h}\left(\alpha P_{p}(g(\nu))+N_{0}\right)\right), & w_{1}^{*}(\nu)>0 \\
& q^{*}(\nu)=0 \\
& u^{*}(\nu)>0
\end{array}\right.
$$

Notice that when $q^{*}(\nu)>0$ and $u^{*}(\nu)=0$, it implies $w_{1}^{*}(\nu) p_{1}^{*}(\nu)+w_{2}^{*}(\nu) p_{2}^{*}(\nu)=\left(w_{1}^{*}(\nu)+w_{2}^{*}(\nu)\right) p_{r p}(\nu)$. Further , if both $p_{1}^{*}(\nu)$ and $p_{2}^{*}(\nu)$ are positive, $A_{1}(\nu)=A_{2}(\nu)=0$ which makes $Z_{1}(\nu)=Z_{2}(\nu)$. So, we can conclude that $p_{1}^{*}(\nu)=p_{2}^{*}(\nu)=p_{r p}(\nu)$. Similarly, when $q^{*}(\nu)=0$ and $u^{*}(\nu)>0, p_{1}^{*}(\nu)=p_{3}^{*}(\nu)=p_{r s}(\nu)$.

Moreover, notice that when $q^{*}(\nu)>0$ and $u^{*}(\nu)=0$, it implies that $p_{1}^{*}(\nu)=\left(\frac{1}{\lambda^{*}+q^{*}(\nu)}-\frac{\alpha P_{p}(g(\nu))+N_{0}}{h}\right)^{+}=$ $p_{r p}(\nu)<p_{w f}\left(\nu, \lambda^{*}\right)$. When $q^{*}(\nu)=0$ and $u^{*}(\nu)>0$, it means $p_{1}^{*}(\nu)=\left(\frac{1}{\lambda^{*}-u^{*}(\nu)}-\frac{\alpha P_{p}(g(\nu))+N_{0}}{h}\right)^{+}=p_{r s}(\nu)>$ $p_{w f}\left(\nu, \lambda^{*}\right)$. Thus, it is impossible to use $p_{1}^{*}(\nu)=p_{r p}(\nu)$ when $p_{w f}\left(\nu, \lambda^{*}\right)<p_{r p}(\nu)$ and $p_{1}^{*}(\nu)=p_{r s}(\nu)$ when $p_{w f}\left(\nu, \lambda^{*}\right)>p_{r s}(\nu)$. So,

$$
p_{1}^{*}(\nu)= \begin{cases}p_{w f}\left(\nu, \lambda^{*}\right), & w_{1}^{*}(\nu)>0, \\ & p_{r s}(\nu)<p_{w f}\left(\nu, \lambda^{*}\right)<p_{r p}(\nu) \\ p_{r p}(\nu), & w_{1}^{*}(\nu)>0, \\ & p_{r p}(\nu)<p_{w f}\left(\nu, \lambda^{*}\right), \\ & p_{r s}(\nu)<p_{w f}\left(\nu, \lambda^{*}\right) \\ p_{r s}(\nu), & w_{1}^{*}(\nu)>0 \\ & p_{r s}(\nu)>p_{w f}\left(\nu, \lambda^{*}\right), \\ & p_{r p}(\nu)>p_{w f}\left(\nu, \lambda^{*}\right)\end{cases}
$$

Therefore if $p_{1}^{*}(\nu)$ exists, it can be written as

$$
\begin{aligned}
p_{1}^{*}(\nu)= & {\left[p_{r s}(\nu)-p_{w f}\left(\nu, \lambda^{*}\right)\right]^{+}-\left[p_{w f}\left(\nu, \lambda^{*}\right)-p_{r p}(\nu)\right]^{+} } \\
& +p_{w f}\left(\nu, \lambda^{*}\right)
\end{aligned}
$$

Similarly, $p_{2}^{*}(\nu)$ to $p_{4}^{*}(\nu)$ can be obtained as follows.

$$
\begin{gathered}
p_{2}^{*}(\nu)=p_{w f}\left(\nu, \lambda^{*}\right)-\left[p_{w f}\left(\nu, \lambda^{*}\right)-p_{r p}(\nu)\right]^{+} \\
p_{3}^{*}(\nu)=p_{w f}\left(\nu, \lambda^{*}\right)+\left[p_{r s}(\nu)-p_{w f}\left(\nu, \lambda^{*}\right)\right]^{+} \\
p_{4}^{*}(\nu)=p_{w f}\left(\nu, \lambda^{*}\right)
\end{gathered}
$$

By KKT conditions and benefit functions defined in (18), we can solve for $w_{k}^{*}(\nu)$ for all $k$ as follows:

$$
w_{k}^{*}(\nu) \begin{cases}=0, & \mathcal{B}_{k, \nu} \leq \Gamma^{*}(\nu) \\ >0, & \mathcal{B}_{k, \nu}=\Gamma^{*}(\nu)\end{cases}
$$

That turns out to be the following consequence.

$$
w_{i}^{*}(\nu)= \begin{cases}1, & \mathcal{B}_{i, \nu}>\max _{i \neq j} \mathcal{B}_{j, \nu} \\ \kappa_{i}^{*}(\nu), & \mathcal{B}_{i, \nu}=\max _{i \neq j} \mathcal{B}_{j, \nu} \\ 0, & \mathcal{B}_{i, \nu}<\max _{i \neq j} \mathcal{B}_{j, \nu}\end{cases}
$$

where $\quad 0<\kappa_{i}^{*}(\nu)<1$

This also explains why $\mathcal{B}_{i, \nu}$ represents a 'benefit function' since the SU will select the highest profit $\mathcal{B}_{i, \nu}=\Gamma^{*}(\nu)$. Moreover, in the case of continuous channel distribution functions $F(\nu)$, the condition $\left(\mathcal{B}_{i, \nu}=\mathcal{B}_{j, \nu}=\Gamma^{*}(\nu)\right)$ is satisfied with zero probability. So, only one $w_{i}^{*}(\nu)=1$ whereas $w_{j}^{*}(\nu)=0, j \neq i$.

\section{REFERENCES}

[1] G. Staple and K. Werbach, "The end of spectrum scarcity," IEEE Spectrum, vol. 41, pp. 48-52, March 2004.

[2] A. Goldsmith, S. Jafar, I. Marić, and S. Srinivasa, "Breaking spectrum gridlock with cognitive radios: An information theoretic perspective," Proceedings of IEEE, vol. 97, pp. 894 - 914, May 2009.

[3] S. Haykin, "Cognitive radio: brain-empowered wireless communications," IEEE journal on selected areas in communications, vol. 23, pp. 201-220, Feb. 2005.

[4] A. Ghasemi and E. S. Sousa, "Fundamental limits of spectrum-sharing in fading environments," IEEE Transactions on Wireless Communications, vol. 6, pp. 649-658, Feb. 2007.

[5] L. Musavian and S. Aïssa, "Capacity and power allocation for spectrumsharing communication in fading channels," IEEE Transactions on Wireless Communications, vol. 8, pp. 148-156, Jan. 2009.

[6] X. Kang, Y.-C. Liang, A. Nallanathan, H. Garg, and R. Zhang, "Optimal power allocation for fading channels in cognitive radio networks: Ergodic capacity and outage capacity," IEEE Transactions on Wireless Communications, vol. 8, pp. 940-950, Feb. 2009.

[7] K. Eswaran, M. Gastpar, and K. Ramchandran, "Bits through ARQs: spectrum sharing with a primary packet system," in IEEE International Symposium on Information Theory (ISIT), (Nice , France), pp. 21712175, June 2007.

[8] G. Ganesan and Y. Li, "Cooperative spectrum sensing in cognitive radio networks," in IEEE International Symposium on New Frontiers in Dynamic Spectrum Access Networks (DySPAN), (Baltimore, MD, USA), pp. 137 - 143, Nov 2005.

[9] R. Zhang, "Optimal power control over fading cognitive radio channels by exploiting primary user CSI," in Proc. IEEE Globecom 2008, (New Orleans, USA), pp. 1-5, Nov. 2008.

[10] X.Kang, R.Zhang, Y.-C. Liang, and H. Garg, "Optimal power allocation for cognitive radio under primary user's outage loss constraint," in IEEE International Conference on Communications, (Dresden, Germany), pp. 1 - 5, June 14-18, 2009.

[11] X.Kang, R.Zhang, Y.-C. Liang, and H. Garg, "On outage capacity of secondary users in fading cognitive radio networks with primary users outage constraint," in IEEE GLOBECOM, (Hawaii, USA), pp. 1 - 5, Nov. 2009.

[12] J. Luo, L. Lin, R.Yates, and P. Spasojevic, "Service outage based power and rate allocation," IEEE Transactions on Information Theory, vol. 49, pp. 323-330, Jan. 2003.

[13] G. Caire, G. Taricco, and E. Biglieri, "Optimum power control over fading channels," IEEE Transactions on Information Theory, vol. 5, pp. 1468-1489, July 1999 .

[14] A. Limmanee, S.Dey, and J. Evans, "Service-outage capacity maximization in cognitive radio," 2010. Technical report [Online] Available at: http://www.cubinlab.ee.unimelb.edu.au/ $\sim$ athipat1/documents/cog_servoutage_rev_new.pdf.

[15] A. Goldsmith and P. Varaiya, "Capacity of fading channel with channel side information," IEEE Transactions on Wireless Communications, vol. 43, pp. 1986-1992, Nov. 1995.

[16] J. Luo, R.Yates, and P. Spasojevic, "Service outage based power and rate allocation for parallel fading channels," IEEE Transactions on Information Theory, vol. 51, no. 7, pp. $2594-2611,2005$. 